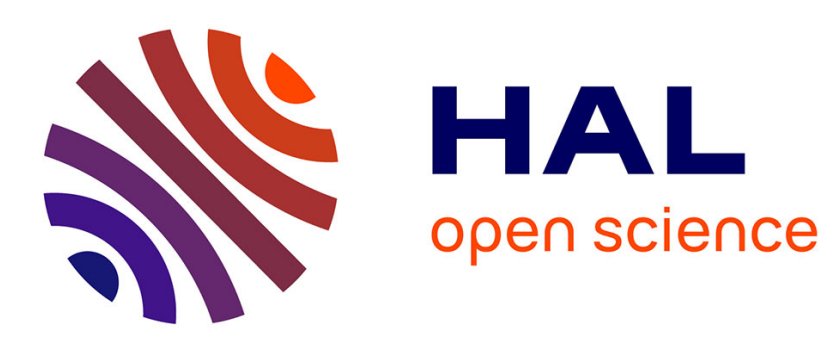

\title{
L'élève et son lycée: De l'espace scolaire aux constructions des territoires lycéens
}

Anne Sgard, André-Frédéric Hoyaux

\section{To cite this version:}

Anne Sgard, André-Frédéric Hoyaux. L'élève et son lycée: De l'espace scolaire aux constructions des territoires lycéens. L'Information géographique, 2006, 70 (3), pp.87-106. 10.3917/lig.703.0087 . halshs-00384440

\section{HAL Id: halshs-00384440 \\ https://shs.hal.science/halshs-00384440}

Submitted on 15 May 2009

HAL is a multi-disciplinary open access archive for the deposit and dissemination of scientific research documents, whether they are published or not. The documents may come from teaching and research institutions in France or abroad, or from public or private research centers.
L'archive ouverte pluridisciplinaire HAL, est destinée au dépôt et à la diffusion de documents scientifiques de niveau recherche, publiés ou non, émanant des établissements d'enseignement et de recherche français ou étrangers, des laboratoires publics ou privés. 


\title{
L'élève et son lycée : De l'espace scolaire aux constructions des territoires lycéens
}

\author{
Anne Sgard et André-Frédéric Hoyaux \\ Groupe de Recherche en Didactique de la Géographie \\ Laboratoire Territoires - UMR PACTE 5194 - Grenoble ${ }^{1}$
}

\begin{abstract}
Résumé :
A la suite d'une série d'entretiens auprès d'élèves pratiquant ou ayant pratiqué le Lycée Edouard Herriot de Voiron (Isère-France), les auteurs analysent le processus de territorialisation et de socialisation des adolescents au sein de l'espace scolaire et s'interrogent sur la pertinence de réfléchir l'établissement en tant qu'espace clos et communauté spécifique d'éducation. Il ressort de l'interprétation des discours produits par les élèves que leurs constructions tendent à effacer la matérialité des délimitations fonctionnelles de l'établissement scolaire pour configurer un territoire scolaire particulier à chacun d'entre eux. Territoire, qui, sill conserve la fonctionnalité des limites, les outrepasse pour les re-construire aux lisières plus ou moins distantes de l'établissement mais cette fois dotées de valeurs positives d'appropriation et d'appartenance. Ces analyses permettent aux auteurs de proposer de nouvelles orientations pédagogiques et une nouvelle réflexion sur le cadre éducatif, notamment du point de vue de la violence scolaire, qui doit se lire à l'aune de la possibilité d'une pleine intégration des adolescents au sein d'un territoire lycéen.
\end{abstract}

\section{Mots-clés :}

Etablissement scolaire, construction territoriale, espace collectif, espace commun, territoire, didactique.

Les établissements scolaires découpent dans les villes, les banlieues, les campagnes, des portions d'espace dévolues à l'accueil et à la formation des enfants et des adolescents. Ceux-ci y passent de longues heures ; jusqu'à atteindre l'âge adulte, leur école demeure l'un des principaux lieux de vie, juste après le domicile familial, où ils découvrent le travail et le jeu, la contrainte et la convivialité, l'ennui et l'excitation, l'amitié et le conflit...

L'école, en tant qu'institution, en tant que communauté spécifique d'apprentissage et de socialisation a été abondamment étudiée par les spécialistes des sciences de l'éducation, mais aussi par les sociologues (Dubet F., 1991, Duru-Bellat M. et Van Zanten A., 1999), les psychologues (Debarbieux E., 2000) et les politologues (Roché S., 1996). Cependant, elle n'a

\footnotetext{
${ }^{1}$ Cet article doit beaucoup aux travaux techniques, aux apports intellectuels et aux relectures critiques des autres membres du Groupe de Recherche en Didactique de Grenoble : Béatrice Vincent (Lycée Edouard Herriot, Voiron), Marie-Alberte Macari (Lycée Pierre Béghin, Moirans), Sonia Chardonnel (Chargé de Recherches - Laboratoire Territoires - UMR PACTE - Grenoble) et Jean David (Responsable du Groupe de Recherche).
} 
pas encore attiré l'attention des géographes, selon une entrée spécifiquement spatiale à partir d'une analyse à grande échelle. Si de nombreux géographes se sont intéressés à l'école, que ce soit à travers une approche démographique (Herin R., Rouault R. et Veschambre V., 1994), socio-politique ou purement didactique (André Y., 1989a ; Chevalier J.-P., 1997 ; Giraud B., 1989 ; Partoune C., 1999), rares sont les auteurs (Bouju A., 1997) qui font référence à l'école en tant qu'espace spécifique, et porteur d'éléments symboliques structurant l'identité territoriale de ceux qui la pratiquent. $\mathrm{Ce}$ désintérêt semble renforcé par les réticences des géographes à analyser et comprendre d'une manière générale les relations qu'entretiennent les enfants et les adolescents à l'espace. Ces derniers apparaissent comme un groupe flou, insaisissable et en constant devenir.

Si quelques travaux fondateurs de la géographie ayant recours aux représentations éclairent quelquefois les relations à l'espace construites par l'élève, celles-ci demeurent ancrées sur la conceptualisation d'objets géographiques: la ville (Audigier F., 1990 ; Biache C., 1997 ; Cavallini V., 1997 ; Clerc P., 1997 ; Guérin M.-C., 1997, Lugadet-Agraz J.B., 1989), la montagne (Masson M., 1995), la région (André Y., 1989b), le globe terrestre (Saarinen T., 1989). Seuls quelques psychologues et anthropologues de l'espace se sont intéressés aux relations entre les pratiques de l'espace et la construction sociale et spatiale de l'enfant et de l'adolescent mais dans des contextes spatiaux différents (Calogirou C., 1991 ; Chombart de Lauwe M.-J., 1987 ; Moles A. et Rohmer E., 1978 ; Piaget J. et Inhelder B., 1948) ou au sein d'un espace restreint de l'établissement scolaire (Delalande J., 2001 ; 2003 ; Derouet-Besson M.-C., 1998).

Pourtant, l'espace scolaire, que l'on peut dans un premier temps approcher comme l'espace géré par l'établissement scolaire, révèle une grande originalité, une étonnante complexité derrière son apparente banalité. Le regard du géographe attaché à l'espace, aux pratiques et aux représentations peut apporter des outils de réflexion et d'intervention à tous les acteurs du système scolaire; c'est en tout cas la conviction du groupe qui s'est penché depuis quelques années sur cet objet de recherche ${ }^{2}$.

En cela, nous avons voulu nous émanciper d'une forme d'autocensure qui tendrait à laisser l'étude des adolescents aux psychologues et aux psychopédagogues. Il était en effet intéressant de reconnaître ces jeunes comme de véritables acteurs territorialisant le monde et sur lesquels il est possible de porter un regard de géographe avec notre vocabulaire, nos outils et nos méthodes.

Il s'agissait donc d'élaborer une analyse à partir des acteurs dans leurs relations à l'espace, autour d'une question centrale : y a-t-il construction symbolique d'un territoire par les adolescents, abordés en tant qu'élèves, dans leur vie d'élèves ? Et quelle est dans cette construction la place accordée au lycée ? La démarche se voulait exploratoire, elle cherchait à ouvrir des pistes, tout en s'efforçant aussi de concevoir des méthodes adaptées de collecte et d'interprétation.

Dans un premier temps, nous avons élaboré une caractérisation a priori de l'espace scolaire, bâtissant une grille de lecture préalable, pour tenter dans un second temps, à partir du matériau constitué par le discours de l'élève, cet usager intensif mais éphémère, d'en démêler les composantes, les usages, les codes et les règles. L'objectif était de comprendre en quoi le lycée, pour les élèves que nous avons observés, constitue un cadre, un matériau et un outil pour un apprentissage de l'espace : espace à construire, à partager, à négocier.

\footnotetext{
${ }^{2}$ Cette étude spécifiquement centrée sur un établissement a été précédée d'un travail de repérage et de problématisation durant deux ans de séminaires : observations sur le terrain, rencontres avec des principaux et des proviseurs, des conseillers d'éducation, ou des architectes spécialisés. Elle a donné lieu à l'élaboration de travaux de Maî́trise à l'Institut de Géographie Alpine.
} 
Au-delà du cadre du lycée, il s'agissait d'étudier la construction quotidienne de la réalité territoriale des adolescents, dans cette étape transitoire mais souvent décisive de leur territorialisation et de leur socialisation (Tap P. et Malewska-Peyre H., 1991) au sein du vaste monde. C'est donc dans l'optique de saisir les pratiques ordinaires des élèves que nous avons récolté notre matériau. Il a été constitué à partir d'un ensemble d'entretiens semidirectifs réalisés auprès de lycéens et d'étudiants ${ }^{3}$ dont le discours se nourrissait d'expériences actuelles et/ou plus anciennes ${ }^{4}$. En effet, si ces discours étaient construits pour partie par l'actualité des pratiques, ils traduisaient également des rapports complexes avec les souvenirs et les projets des uns et des autres. Souvenirs et projets ancrés plus ou moins loin du contexte d'entretien.

Pour aborder la thématique de l'élève dans l'espace scolaire, nous avons utilisé dans un premier temps une approche extérieure, distanciée, visant à observer, à manipuler cet objet encore mal défini pour en isoler la spécificité et nous fournir une première grille de lecture faisant prise pour notre questionnement. Dans un second temps, nous avons confronté l'a priori de cette construction problématique sur l'espace scolaire avec les constructions symboliques des territoire(s) scolaire(s) nées des discours collectés auprès des élèves et étudiants.

\section{L'espace scolaire, un espace fragmenté et difficilement partagé.}

Si l'on tente dans un premier temps d'identifier les caractères originaux de l'espace scolaire, de l'école maternelle au lycée, un ensemble de traits se dégagent:

- Tout d'abord, c'est un espace fortement spécifié, immédiatement reconnaissable de l'extérieur, au-delà des évolutions architecturales qui accompagnent l'histoire de l'institution, avec les éléments propres que sont la cour, le préau, les grands bâtiments abritant les salles aux larges fenêtres, les parkings. Dès le plus jeune âge, celui qui y pénètre, reconnaît immanquablement les salles de classe, les bureaux administratifs, la bibliothèque, le CDI, la cantine.

- Si l'espace est facilement identifiable dans sa globalité, il n'en est pas moins complexe dans son organisation interne. Quelle que soit sa nature, école, collège ou lycée, l'espace scolaire est fragmenté à l'extrême : d'un bâtiment à l'autre, d'une salle à l'autre, d'un couloir à un vestibule, les fonctions et les utilisateurs varient constamment, et en parallèle les règles d'usage, souvent implicites, sont à chaque fois modifiées.

- A cette fragmentation répond des changements d'ambiance : ici le bruit de la cour de récréation, là le silence de la bibliothèque ; le décor intérieur, les matériaux, le mobilier, la lumière sont autant de signaux envoyés à l'utilisateur des lieux. Certaines portions d'espace sont réservées aux élèves, ailleurs ils sont interdits, au mieux tolérés.

- Cette fragmentation spatiale répond à une fragmentation sociale et culturelle, qui confine au cloisonnement. Ce que l'on appelle volontiers la communauté éducative est constituée d'une grande diversité de membres aux pratiques et aux temporalités fortement différenciées, qui usent chacun à leur façon de l'espace. Deux exemples peuvent l'illustrer : les enseignants se partagent entre les salles de cours et leur «salle des profs», et

\footnotetext{
${ }^{3}$ Une vingtaine d'entretiens semi-directifs ont été menés auprès d'un groupe d'élèves de seconde et de première du Lycée Edouard Herriot de Voiron (Isère) et d'un groupe d'étudiants de DEUG et de Licence d'Histoire à l'université $P$. Mendès-France de Grenoble, anciens élèves de ce même lycée. Ce lycée est un établissement d'enseignement général, d'environ 1600 élèves, situé dans une ville moyenne, Voiron, à une vingtaine de kilomètres de Grenoble. Il peut être qualifié de « gros » lycée, au vu de ses effectifs, et «sans problème » de l'avis des élèves, enseignants et administratifs. L'origine des élèves se partage entre la ville de Voiron et une vaste zone périurbaine et rurale aux marges de la métropole grenobloise.

${ }^{4}$ Deux protocoles d'entretien ont donc été élaborés. Néanmoins le matériau principal est constitué par les entretiens avec les lycéens, les témoignages des étudiants venant en guise de prolongement ponctuel.
} 
fréquentent aussi occasionnellement la cour, la documentation, la cantine, les bureaux administratifs ; ils sont présents une partie de la semaine, leur journée rythmée par la succession des heures de cours, et demeurent dans le même établissement pendant des durées extrêmement diverses: certains pour un bref remplacement, d'autres pour de longues années. Le personnel administratif, quant à lui, au rythme de fréquentation beaucoup plus régulier et sur des durées de poste généralement plus longues, limite en revanche son usage de l'espace à des portions réduites, les bureaux, peu en contact avec les élèves.

On peut ainsi identifier un grand nombre de collectifs aux âges, ancienneté, motivation, implication diverses qui cohabitent, apportant chacun une culture professionnelle différente et une représentation de l'institution scolaire et de sa propre place dans cette institution. Sans aller au-delà dans cette analyse qui déborde largement le cadre de notre démarche, on retiendra qu'à la diversité des statuts et des fonctions répond la diversité des usages des lieux. On relèvera aussi l'imbrication constante des pratiques spatiales et des temporalités, élément clé de notre approche.

Car si l'espace de l'établissement scolaire se veut un espace collectif, il est cependant difficilement partagé. Pour contrecarrer cette fragmentation, des règles d'usage des lieux et des temps y sont imposées, elles y font tout d'abord œuvre de socialisation puisqu' «à l'occasion d'expériences éducatives et grâce à la médiation d'agents sociaux significatifs », l'élève « doit intégrer ces données sociales et culturelles à la structure de sa personnalité ». En ce sens, le système éducatif " sert non seulement à transmettre les exigences et les acquis sociaux, mais aussi à perpétuer la société » (Tap P., 1991, 49). En cela, notre double approche par l'espace et le temps prend toute son importance puisque les lieux et les rythmes sont bien les repères structurants de toute socialisation, de tout apprentissage de la collectivité, mais aussi l'ébauche de toute territorialisation du monde. La mise en place de ces repères structurants configure alors un espace-temps supposé des contraintes et un espace-temps supposé des libertés qui prédéterminent en quelque sorte toute mise en situation sociale et spatiale au sein de l'établissement scolaire (Senore D., 2000).

La complexité de l'espace scolaire tient en grande partie à la multiplication des règles d'usage attachées à ces différents lieux, règles tour à tour écrites ou orales, revendiquées, invoquées, ou implicites, toujours variables. Dans cet espace conçu pour les élèves, ce sont finalement les adultes qui sont seuls détenteurs des règles : le même lieu peut changer de règle simplement parce que l'adulte a changé ; à l'élève de s'adapter, c'est-à-dire souvent, comme nous le verrons par la suite, de la détourner ou de se la réapproprier. Chaque enseignant dans sa salle impose à ses élèves des règles de conduite qui s'effacent dès que le seuil de la salle de cours est franchi : bruit, déplacements, choix de la place, l'usage du lieu est multiple et sans cesse réajusté : il n'y a donc pas d' « unité de lieu » (Roché S., 2000, 75).

L'omniprésence de la règle va de pair avec un mode d'énonciation qui reste le plus souvent du domaine de l'implicite ou de l'improvisation. L'élève ne sait pas le jour de la rentrée quelles sont les règles de conduite de chaque adulte auquel il aura affaire. Ces règles particulières s'imbriquent et se surimposent dans un établissement lui-même régi par un règlement collectif, le seul qui soit écrit et connu de tous. Connu mais pas forcément suivi puisque toute règle inscrit inévitablement l'assentiment ou l'opposition des uns et des autres qui par là-même se positionnent au travers elle en tant qu'acteur et envers les autres acteurs. Ainsi, au sein même de cet espace normé, certains lieux sont fréquemment le théâtre de transgressions plus ou moins explicites (que ce soit d'ailleurs par les élèves ou... les professeurs). Ce sont le plus souvent des lieux en périphérie (ordre spatial) ou de transition (ordre temporel) par rapport aux activités du travail lui-même et dont la connotation relève plus du délassement que de l'obéissance : couloirs, escaliers, vestiaires et 
toilettes. Cette imbrication construit progressivement une norme collective, largement implicite, faite d'éléments particuliers, qui ont la durée d'existence de celui qui l'impose - un enseignant, un directeur - et d'éléments collectifs qui se pérennisent et caractérisent l'ensemble de l'établissement et ceux qui y cohabitent.

Quand l'élève arrive dans son établissement, il pénètre donc conjointement un espace totalement organisé, laissant peu de place au changement, une institution elle aussi fortement structurée et un temps avant tout caractérisé par la contrainte : heures d'entrée et de sortie, emplois du temps, le temps scolaire et ses métriques sont donc omniprésents. Pour l'enfant qui rentre à l'école maternelle, cet espace-temps est souvent le premier qu'il découvre en dehors du milieu familial, et il constitue sa première expérience d'un espace social à la fois réglé et partagé : rester à sa place et respecter celle des autres, ne pas sortir d'une salle, se déplacer selon certains itinéraires, organiser ses cheminements dans la cour, éviter les lieux interdits. Cette expérience est simultanément et de manière totalement imbriquée celle du temps social : c'est la journée d'école qu'il va falloir suivre dans sa totalité, le rythme imposé des activités, le temps du jeu et le temps du travail, les sonneries ! L'école est le lieu de l'apprentissage scolaire des notions d'espace et de temps, c'est aussi le lieu de l'apprentissage social de l'espace partagé et du temps collectif. « Tous les enfants de tous les milieux sociaux sont confrontés à l' «apprentissage » de la spécificité et de la diversité des temps et des temporalités qui rythment la séquence « du réveil à la cloche », écrit une sociologue étudiant les «temps quotidiens » des plus jeunes (Haicault M., 1989). Pour le lycéen, l'apprentissage se prolonge à travers un nouveau cadre : plus vaste mais mieux maîtrisé, plus souple vis à vis des pratiques adolescentes mais toujours contraignant dans son organisation et ses rythmes.

De ce fait, l'espace scolaire peut être au mieux caractérisé d' « espace collectif ». Car s'il est « public » au sens juridique du terme et «commun » dans la mesure où il «permet la coprésence des acteurs sociaux, sortis de leur cadre domestique » (Lussault M., 2003, 334), il demeure cependant un espace réservé, clos. Il est en outre un espace de présence obligatoire, normé dans ses fonctions et les pratiques qui doivent s'y engager.

Il n'est donc pas public au sens où il n'est pas accessible à tous. L'identité des membres de la « communauté éducative » qu'il accueille est strictement définie et limitée, et la tendance actuelle est à la fermeture de plus en plus hermétique et à la surveillance étroite des limites. Il n'est pas non plus commun dans la mesure où le suivi des apprentissages par les élèves est loin de relever toujours d'un projet intentionnel. L'espace scolaire se caractérise par la contrainte qui amène les acteurs principaux, les élèves, selon des rythmes et dans des lieux imposés, à se plier à un système normatif sur lequel ils ont peu de prise. Ainsi, dans l'espace scolaire, l'élève suit des règles, alors que dans l'espace commun (une discothèque par exemple) les acteurs convergent intentionnellement pour pratiquer des actes ludiques plus ou moins normés mais préalablement et intersubjectivement établis, voulus et acceptés.

Dès lors, pendant sa vie d'élève, l'enfant puis l'adolescent, intègre des durées, rythmes et cycles, s'approprie un espace qu'il finit par connaître parfaitement, et l'espacetemps de l'école devient l'un des pôles organisateurs de ses pratiques quotidiennes, de sa temporalité et, vraisemblablement, de sa territorialité toujours en construction. Ces pratiques en partie surdéterminées par l'espace scolaire ne limite pas pour autant le champ de l'appréhension du monde de l'élève et ipso facto sa construction territoriale. D'une part, parce qu'au sein même de l'espace scolaire, le poids des règles, des rythmes et des métriques n'agit pas de la même manière chez tous les élèves. D'autre part, parce que chaque élève possède d'autres champs d'investigation spatiaux, sociaux et temporels dans son existence quotidienne. 
Après l'appréhension de l'espace scolaire de manière objectivante, notre intérêt s'est donc déplacé vers l'analyse du discours de quelques élèves sur leur lycée. L'objectif était triple : d'une part, mieux comprendre les lignes de force de leur lecture de l'espace scolaire en tant qu'espace aménagé, construit ; d'autre part mieux appréhender le poids des règles et normes implicites de l'espace scolaire en tant qu'elles sont médiatrices de la représentation et de la pratique de cet espace ; enfin, élucider les codes qu'ils construisent individuellement ou collectivement pour s'abstraire parfois de ces règles et modifier la structure même de l'espace scolaire dans ses limites. Car si des normes et des règles s'imposent, les élèves en tant qu'ils définissent leur façon d'être acteurs pour eux-mêmes et vis-à-vis des autres, par la territorialisation et la socialisation, disposent d'un panel de pratiques physiques et discursives pour les contrôler. Ce contrôle s'effectue par la possibilité qu'ils ont d'avoir une certaine emprise sur le monde qui les entoure par la mise à distance de ces normes et de ces règles. Cette emprise leur permet de construire leur(s) propre(s) territoire(s) et par là-même leur identité territoriale singulière.

\section{Le bahut : un territoire fait de coins, de marges et de routines}

Le protocole d'entretien avec les élèves propose, selon une succession très précise, un ensemble de questions, ou plutôt de pistes de réflexion, au sein desquelles le lycée n'apparaît que tardivement, après des questions orientées vers le domicile, les pratiques, les itinéraires et lieux fréquentés, et des questions sur la région. L'objectif est d'analyser dans quelle mesure le lycée est mobilisé spontanément par les élèves comme l'un des éléments structurant de leurs pratiques et de leur territorialité. Ce n'est donc qu'en deuxième partie d'entretien que le lycée est abordé délibérément, avec des questions portant sur les modes de fréquentation, sur les lieux et espaces, sur l'architecture. A partir de ce matériau, il s'est agi d'identifier les éléments constitutifs de l'espace scolaire tels que les élèves les disent et en les disant construisent leur territoire scolaire fait d'appropriation, de délimitation, de différenciation.

D'un point de vue méthodologique, il faut noter d'emblée que notre démarche d'entretien restreint quelque peu la collecte: rencontrant des élèves volontaires, dans le cadre d'un lycée «banal, sans problèmes », nous n'avons bien sûr pas touché les élèves en rupture avec le système scolaire; mais notre objectif n'est pas de recueillir la diversité des représentations du lycée, démarche qui justifierait à elle seule un autre programme de recherche.

L'exploitation de ces entretiens ${ }^{5}$ nous a amenés à dégager trois grands thèmes structurant le discours des élèves non pas sur mais à propos du lycée : la qualification de cet espace autour de la dialectique contrainte-convivialité, la question des limites et des marges, l'appropriation différenciée d'un espace-temps.

\section{Contraintes et convivialité font du lycée un lieu central}

Depuis J. Piaget, l'appréhension de l'espace par les enfants et les adolescents a été largement étudiée, il ne s'agit donc pas pour nous de contribuer à cet édifice. Notre approche vise l'analyse du discours sur l'espace des adolescents qui, à l'âge lycéen, voient leur périmètre de pratique quotidien s'élargir, avec une mobilité, une autonomie, des modes de déplacement et des marges de liberté accrus. Ces entretiens ont confirmé que les adolescents n'ont pas une vision globale de leur espace de pratique, qu'ils peinent à verbaliser : celui-ci associe de manière lâche des lieux, eux, fortement spécifiés, notamment

\footnotetext{
5 L'exploitation est manuelle, à partir d'une transcription rigoureuse de chaque entretien, d'une part de manière individuelle autour d'une grille de lecture commune, d'autre part de manière transversale.
} 
autour de critères affectifs et de convivialité. Leur discours porte avant tout sur des relations sociales ou des pratiques collectives, avec leur famille ou leurs copains ; les lieux sont dès lors rattachés à des activités, des échanges, des rencontres, et non pas dotés de caractères spatiaux, architecturaux ou esthétiques. Seuls les adolescents habitant en périphérie ou utilisant un mode de déplacement propre (mobylette, scooter) identifient des itinéraires et des points de repère répondant immédiatement à leur besoin de mobilité.

« Pour aller en cours, j'prends le car à la place, ça fait quatre kilomètres de chez moi, c'est ma maman qui m'emmène jusque là-bas et après ben on part, on prend la direction de Saint-Joseph de Rivière, après Saint-Etienne de Crosset puis après ben Voiron, on descend à la cathédrale et puis on monte directement au lycée » (Elève de Seconde)

Il n'en découle cependant pas forcément une connaissance globale d'un espace à plus petite échelle dont ils pourraient tracer une carte, mais plutôt des liens, des lignes de forces reliant des points vaguement situés que seraient les lieux d'activité principaux (lieu de résidence, lycée, lieu( $\mathrm{x}$ ) de pratique sportive, lieu( $\mathrm{x}$ ) de chalandise) formant un itinéraire, une sorte de réseau polarisé mal articulé.

« La Maison-Réaumont pour aller voir mon cheval. Le lycée. Rives pour aller faire les courses, les trucs comme ça. Si c'est les gros trucs, on va sur Voiron. Je joue au basket à Apprieu » (Elève de Première)

De la même manière, nous avons cherché en vain une appréciation d'ordre esthétique sur le lycée ou son parc : nos questions apparaissaient manifestement incongrues à nos interlocuteurs qui fournissaient des réponses rapides et convenues sur la beauté des arbres ou le vert des pelouses, voire sur la propreté des lieux, notamment l'insalubrité supposée des bâtiments de l'internat en cours de rénovation.

« [Le lycée] est assez agréable, enfin, il est quand même en bon état, il est tout neuf donc c'est quand même agréable. Sinon, l'architecture, elle est commune, elle est ordinaire quoi, pas originale, c'est pas le truc, je l'ai jamais vu quelque part » (Elève de Première)

« J'pense déjà quill y a une grande différence entre linternat et l'externat. L'internat, il est un peu vieux quoi. Y'a certains endroits où ça se voit bien et c'est pas le même entretien du tout. Ici, ils s'en occupent bien, ils font attention à la propreté et tout alors qu'à l'internat on a limpression qu'y a rien de fait quoi. Cet hiver quand il a plu vraiment beaucoup, y'avait des trous au plafond et y'avait de l'eau qui coulait ».(Elève de Seconde)

Il sera difficile dans ces conditions de parler d'espace à propos des élèves : leur vocabulaire use très peu de termes spatiaux et leur mode de repérage et d'organisation élude l'espace autant que possible, si ce n'est pour parler de l'aspect imposant du bâtiment, ainsi que la taille des classes qui marque l'élève et cela d'autant plus s'il vient d'y entrer (notamment les Secondes). Aspect renforcé encore dans cet établissement par la mise en scène des bâtiments à flanc de collines.

«C'est quand même des bâtiments imposants. C'est grand, et on a beaucoup d'espace quand on est à l'intérieur. On a de grandes classes. Quand j'ai vu le bâtiment, $j^{\prime}$ ai dit : ouh la la mais après on s'y repère vite » (Elève de Seconde)

Cette place accordée à l'usage des lieux, aux pratiques localisées, permet cependant d'engager une analyse en termes d'appropriation, d'attachement ou de rejet de cet espace, c'est-à-dire d'engager une réflexion sur la mise en configuration de l'espace par l'élève, autrement dit sa territorialisation du monde.

L'expression configuration nous paraît ici pertinente car elle permet d'articuler, à travers la dimension subjective, affective et symbolique du discours, les différents éléments matériels - essentiellement des objets dans les lieux - au sein d'un monde fait de multiples 
territoires plus ou moins vastes, plus ou moins délimités, et plus ou moins clairement définis dans leur substance et dans les relations qui s'y déroulent. En ce sens, l'élève configure l'image qu'il se fait de l'espace. Cette configuration est une construction de sa réalité vécue de l'espace, par une territorialisation et une socialisation du monde qu'exprime en partie l'acte de parole.

Ce que nous interprétons des entretiens, ce sont donc des configurations associant lieux et rares itinéraires, gommant les distances, rejetant au « loin » des lieux négatifs, pour en charger d'autres de sens et de sentiments et les ramener du coup «à leur portée » (Hoyaux A.-F., 2003). Cette mise à portée des lieux qu'ils s'approprient élabore une juxtaposition de territoires plus ou moins éloignés du lieu d'entretien et l'on a parfois l'impression de passer du « coq à l'âne spatial ».

«J'sais que quand je vais au coin fumeur enfin ici à la cour du gymnase et ben c'est toujours au même banc, toujours au même endroit, $j$ 'sais pas, on fait pas attention. Ben sinon, à Saint-Laurent, c'est le stade donc on est tous là-bas donc on fait pas attention mais le territoire personnel, c'est ma chambre quoi » (Elève de Seconde)

Dans ces configurations, le lycée occupe une place centrale, de manière objective par l'intensité de la fréquentation et aussi par le rôle structurant dans la sociabilité des élèves : le lycée est le lieu par excellence d'expérimentation des relations avec les autres adolescents et avec les adultes. Le lycée est du coup toujours présent, ambivalent, qualifié parfois de manière contradictoire entre le discours convenu de dénigrement et des remarques au fil de l'entretien où il apparaît que le «bahut » n'est pas si désagréable que ça. Quand ils le comparent au lycée le plus proche, c'est toujours en des termes positifs, liés à la situation urbaine et centrale du lycée ${ }^{6}$.

« ben oui, moi j'trouve que ça va hein, comparé à d'autres, ça va, c'est plutôt joli » (Elève de Seconde)

C'est du reste une constante chez tous les élèves de nommer leur lycée tantôt par un terme d'argot générique : bahut, caisson... largement emprunté au vocabulaire pénitentiaire, même pour des établissements récents dont l'architecture n'a que peu de similitude avec celle des prisons du XIXème siècle! Généralement les élèves usent également d'un surnom propre à l'établissement : diminutif très souvent, sigle, nom en « verlant » transmis de génération en génération.

Dans le cas de notre enquête, ce nom dépend tout d'abord de l'indexation à la situation sociale établie entre l'élève et son interlocuteur. A la question «comment nommes-tu le lycée »? :

« ben, ça dépend avec qui ! Si c'est avec des personnes que je suis tout le temps, c'est le bahut ou alors c'est Edouard avec des gens qui sont pas du lycée, et ils savent tout de suite quoi $\gg$ (Elève de Seconde)

« Je suis dans le Lycée Edouard Herriot, où le Lycée Edouard ou à Edouard ouais, ça dépend avec qui je parle si ils ne connaissent pas. Avec mes copines, le bahut» (Elève de Première)

A Voiron, les élèves appellent le lycée Edouard Herriot, tout d'abord indistinctement et encore longtemps après l'avoir quitté : Edouard. Le géographe attentif à la toponymie, ne manquera pas de voir dans cette vieille habitude la marque d'une appropriation mais aussi un « patrimoine » à verser à la culture et à l'image de marque de l'établissement. En ce sens, l'appartenance à un lycée constitue l'un des éléments principaux de la «carte

\footnotetext{
${ }^{6}$ Cette appréciation se confirme fréquemment auprès des élèves de tous âges (mon école est mieux que les autres) et a donné lieu a de nombreuses expériences d'échanges entre établissements, visant à susciter un sentiment d'appartenance. Le lycée cité par les élèves est le Lycée P. Béghin de Moirans, ville voisine : lycée flambant neuf mais qu'une localisation excentrée, éloignée de toute animation urbaine, semble vouer à la disgrâce.
} 
d'identité » de l'élève quand il se trouve en dehors de son cadre habituel: je m'appelle $X$ et je suis à Edouard. Cette appartenance reste aussi présente dans l'identification des étudiants qui se retrouvent volontiers entre anciens, même s'ils ne se fréquentaient pas en tant que lycéens. Il y a une forme de localisation que ne rend pas le terme bahut, pourtant plus usité dans les relations personnelles, car évoquant sans doute davantage l'esprit de classe, celui d'adolescents de mêmes âges, quels que soient les lieux de France et d'ailleurs, qui sont contraints d'aller au lycée.

«On est content de le quitter le week-end. Oui enfin, on est obligé d'y aller donc, mais il faut pas se plaindre, mais bon, c'est clair quand je peux partir je pars même si je respecte le lycée mais c'est le week-end 》 (Elève de Première)

Le lycée apparaît donc dans le discours sur le territoire, omniprésent et ambivalent, autour du binôme contrainte-convivialité. C'est le lieu d'apprentissage de la contrainte spatiale et temporelle, et cela ressort bien des entretiens qui mettent en avant, explicitement ou non, l'organisation du temps et des déplacements autour et en fonction du lycée. En parallèle, le lycée reste le lieu principal où se font et se défont les amitiés, les groupes de toutes tailles, les couples, et cette vie sociale occupe une large part du temps et des préoccupations. Dans les entretiens auprès des étudiants anciens élèves du lycée, ce second élément de la dialectique prédomine largement sur le premier : la contrainte est intégrée, acceptée, parce qu'elle se prolonge de manière atténuée dans les autres cadres de la vie sociale : dès lors, son apprentissage est oublié.

Cette dialectique contrainte-convivialité guide également leur discours sur l'intérieur du lycée, ce que nous abordons dans notre questionnement initial comme un espace fragmenté, difficilement partagé. Cette fragmentation apparaît en filigrane, avant tout selon la modalité absence-présence : les lieux de convivialité ressortent constamment dans les entretiens à travers une description des pratiques qui leur sont attachées, alors que les élèves éludent volontiers les parties du lycée où ils ne se sentent pas à l'aise ou qui leur sont interdites.

Le territoire qui se dessine dans le discours des élèves est, implicitement, très différencié, très compartimenté, en fonction d'impressions désagréables (les salles bruyantes et dégradées, les couloirs trop étroits, un chemin trop raide...) ou de sensations positives (un CDI calme, un banc dans le parc).

« y'a certaines salles, c'est pas rénové et c'est pas le même entretien du tout! Déjà les murs sont tout petits donc on entend tout d'une classe à l'autre, alors que là on est bien installé, j'veux dire, on a du matériel tout neuf, donc tout de suite ça change complètement 》 (Elève de Seconde)

«Si jai un trou, je vais soit en étude soit au CDI, ça dépend si on a pas beaucoup de choses. Si on veut discuter, on va plutôt en étude parce que au CDI, c'est un peu (rires) [...] A l'étude, on peut discuter, on peut faire ce qu'on veut. Des fois aussi, je vais au foyer parce qu'il y a de la musique. Mais ça dépend ce qu'on veut faire et tout, on choisit un peu nos endroits. Au CDI, y'a l'avantage d'y avoir un peu de calme » (Elève de Première)

On voit également les élèves organiser leurs cheminements, leurs pratiques des lieux selon des logiques universelles : évitement, contournement, accaparement (Augoyard J.-F., 1979). Le meilleur exemple est sans doute celui du « coin fumeur » ; ce coin, délimité dans la cour par une simple bordure de ciment, représente le lieu identifié non par une fonction précise ou par un interdit, cas de la plupart des lieux intégrés au lycée, mais par l'autorisation, le droit, on dirait presque la liberté. Il n'a d'autre caractère que celui-là : rectangle de gravier sans équipement particulier, où l'on a officiellement le droit de fumer. Ce coin est présent dans la totalité des entretiens: c'est le coin des élèves, lieu de convivialité par excellence, où tout le monde se retrouve même les non fumeurs. En cela, 
c'est un espace commun dont les désagréments de la fumée sont minimisés par chaque élève au profit de l'intention d'y retrouver ses relations privilégiées. Et cela même si la superficie de ce «coin » ne permet sûrement pas à la totalité des élèves du lycée de s'y retrouver effectivement, en tout cas autant qu'ils semblent nous le dire.'

« [Pourquoi] Y aller même si on fume pas? mais j'sais pas, y'a plus de monde en fait et les gens y ont plus tendance à parler. Enfin, c'est plus convivial. » (Elève de Premère)

« Là où on se rend le plus souvent, c'est la cour du gymnase vu que y'en a certains qui fument et c'est le seul lieu où c'est toléré. [La cour du gymnase ?] On l'appelle comme ça mais nous on dit le coin fumeur » (Elève de Seconde)

L'analyse de ce couple contrainte et convivialité nous permet alors d'étayer plus en profondeur le couple espace commun et espace collectif qui interpelle M. Lussault et J. Lévy dans leurs analyses de l'espace public (2003). En effet, quand le lycée est construit par l'élève sur le principe de la contrainte, il relève exemplairement de l'espace collectif, alors que lorsqu'il est conçu sur le principe de la convivialité, il ressort de l'espace commun. Il nous semble qu'il y a même plus que cela, comme le montre par exemple la nomination affective du Lycée, «Edouard », qui renvoie à une forme d'appropriation que le concept d'espace commun ne rend pas forcément dans toute sa dimension. On peut intentionnellement vouloir se rendre dans un lieu sans pour autant y projeter des attaches affectives tels que ce lieu soit considéré comme participant du territoire de l'élève, de cette référence intime qui est comme un prolongement de celui-ci tant au niveau des relations sociales et spatiales que cette référence intime sous-entend. Par la toponymie usitée, la personnalisation du bahut tend à exprimer ces relations particulières.

Nous pouvons ainsi échelonner trois niveaux d'implication de l'élève par rapport à l'espace scolaire, l'un lié à la contrainte, l'autre à la convivialité, le dernier à l'affectivité, ce qui permet de proposer trois niveaux d'implication dans la relation à l'espace : celle de l'espace collectif, de l'espace commun, et du territoire. Mais si les deux premiers niveaux d'implication s'attachent à des espaces délimités (c'est pour cela qu'il reste de l'ordre de l'espace), le troisième niveau d'implication, par sa caractérisation même de prolongement, amène à réfléchir sur les délimitations fonctionnelles et symboliques qui le définissent. Ces délimitations ne sont plus a priori déterminantes pour les pratiques et les comportements attendus de l'élève (la classe, la cantine) mais construites par ce dernier pour structurer sa personnalité. Il est donc intéressant d'interpréter en quoi les limites de ce territoire ne sont plus rigides mais peuvent être lacunaires, mouvantes, flexibles, et en quoi cela constitue pour partie des arguments à sa territorialisation et à sa socialisation.

\section{Limites effacées et marges mouvantes}

Si l'on progresse encore dans la lecture du lycée tel qu'il est raconté par les élèves, un élément clé de notre grille de lecture initiale a été en effet rapidement bousculé : de la même manière que nous avons dû renoncer au terme d'espace scolaire, nous devons réviser notre approche de l'établissement au sens de bâtiment ou ensemble de bâtiments, découpant une portion d'espace facilement identifiable, et matériellement bien délimitée par les grilles, murs, portails qui sont les repères matériels qui viennent immédiatement à l'esprit.

Le thème des limites et des marges a donc fait irruption dans notre grille d'analyse sous une forme inattendue: son absence du discours de la quasi-totalité des élèves.

\footnotetext{
${ }^{7}$ L'exemple de ce « coin », symboliquement central, est à verser au dossier de la réflexion sur les modalités d'application de la «loi Evin » dans les lycées.
} 
Précisons que les limites matérielles sont tout à fait présentes visuellement autour du lycée étudié, si l'on tente une lecture objective. Le lycée Edouard Herriot dispose d'une vaste superficie, répartie entre plusieurs bâtiments et bordée par un grand parc ; néanmoins le lycée et son parc sont clôturés par des grilles et de hauts murs, l'entrée se fait par un sas, surveillé par vidéo comme c'est le cas de plus en plus fréquemment aujourd'hui, et toute incursion est étroitement surveillée par un gardien vigilant.

De ces limites, de ces murs, de ce portail, aucune trace dans les entretiens des élèves malgré des relances presque insistantes de la part d'enquêteurs eux-mêmes désagréablement impressionnés... Si l'on parle du lycée comme d'un «caisson », on ne le vit pas sous le registre de l'enfermement :

« C'est vrai que si j'ai envie de sortir, et ben je sors. Et puis je re-rentre sans avoir à faire sauter les cours » (Elève de Première)

C'est bien au contraire ce qui ressort comme un des éléments communs aux diverses configurations : oubli des limites, effacement des murs et des grilles, au profit de marges mouvantes qui tendent à étendre le lycée en dehors de ses limites matériellement tracées. Ainsi, la rue longeant l'entrée avec le muret et les bancs où les élèves se retrouvent le matin fait partie du lycée ; dans certains entretiens on a même l'impression que la place où les cars scolaires déversent les cohortes d'élèves chaque matin, voire le car scolaire lui-même, sont également intégrés dans ce que les élèves appellent le lycée.

« Et après ça dépend où sont les amis, y'a le télécom là où y'a le car que prennent certaines personnes et sinon c'est la gare, c'est tout. C'est à peu près tout et puis le lycée, les différents lycées aussi. Y'a les bancs qu'on peut pas louper quoi, comme quand on descend en face du lycée, y'a une route et y'a une sorte de petit monument et y'a des bancs autour donc là ouais souvent, enfin quand on a le temps on s'arrête un moment là » (Elève de Seconde).

« J'vais souvent à la place Saint-Bruno ou alors quand il fait mauvais temps, on va soit boire un coup au Parisien ou au Mégaz mais sinon on reste bien là aussi ou des fois on va se promener dans le parc à Voiron » (Elève de Seconde).

Ces marges extensibles, repoussées par les pratiques, incorporent aussi dans l'objet lycée un lieu essentiel à la vie lycéenne : le bar, lieu de retrouvaille, de convivialité, souvent de travail ; les élèves y attendent le début des cours, s'y retrouvent pendant les interclasses, ou à midi, ou en attendant le car. Tous ont leur bar attitré et si possible leur table, parfois leur chaise; ils expriment clairement cette appropriation du lieu, leur désagrément s'ils trouvent leur table occupée. C'est aussi le lieu le plus précisément qualifié dans les entretiens : bruit, odeur, contacts, lumière.

Les limites suggérées par ces configurations sont donc mouvantes, instables, difficilement lisibles, elles ne s'appuient pas sur les éléments matériels disponibles comme les grilles ou les portes. Plus que des limites séparant des espaces bien identifiés, ce sont des marges ou des interfaces, ouvrant sur des pratiques différentes. Ainsi les bars servent d'interface entre le monde lycéen et la ville, connue, fréquentée occasionnellement mais moins intensément appropriée que le lycée. Alors que la partie de l'établissement dévolue à l'administration est séparée par une barrière, invisible mais efficace dans les pratiques et les discours, vis-à-vis d'un espace que les élèves ignorent et ne cherchent pas à connaître. Pour pousser l'analyse, on pourrait dire que le bar fait parfois davantage partie du lycée que le bureau du proviseur...

\section{Temporalités et routines lycéennes}

Ce qui se dégage clairement des entretiens c'est l'imbrication étroite du temps et de l'espace. Cet élément du questionnement initial s'est révélé pertinent, au-delà même de 
notre attente : le lycée est avant tout pour son usager un espace-temps. C'est aussi en cela que le lycée, dans l'appréhension qu'en a l'élève, devient un de ses territoires. Et si l'espace semble gommé au profit d'une configuration en territoire-archipel, pour reprendre une expression usée mais justifiée ici malgré des réseaux ténus entre les lieux, ce sont les temporalités lycéennes qui apparaissent comme l'élément organisateur de ces configurations. Nous avons insisté sur l'importance de l'école (en général) dans l'apprentissage du temps social : les élèves l'ont confirmé. La journée, la semaine sont rythmées par l'emploi du temps, les ramassages scolaires, dans lesquelles s'imbriquent les activités « para-scolaires ». Ainsi, sans que nous évoquions le moins du monde les rythmes dans nos questions, ces derniers apparaissent ou se révèlent former la trame des divers itinéraires.

«Pour aller en cours, j'prends le car à la place [...] tous les matins. [...] Souvent j'fais du judo donc c'est tous les soirs pratiquement, deux trois fois par semaine [...] sinon je vais chez mes amies le mercredi après-midi [...] sinon ben j'vais souvent chez mon parrain et ma marraine, ils habitent plus loin vers Bourgoin mais $j$ 'y vais pratiquement tous les week-ends » (Elève de Seconde)

Loin de s'en plaindre, voire de l'objectiver, les élèves l'expriment incidemment comme un élément évident de leur vie; ils tendent même à l'accentuer en introduisant dans ce rythme imposé, des routines qu'ils s'inventent et qui viennent combler les moments creux de l'emploi du temps. Ces routines nous apparaissent comme un élément clé de la territorialité des élèves dans la mesure où elles sont rattachées à des lieux précis, à des itinéraires invariables: ainsi la fréquentation du bar conjugue des moments précis avec des places déterminées ; la station sur le muret avant le début des cours relève de ces mêmes routines spatio-temporelles.

« on va souvent au Parisien [...] On garde habituellement le même train train quoi. On essaie de pas trop changer » (Elève de Seconde)

Ce sont ces routines qui amènent à intégrer les marges diffuses dans ce que les élèves appellent le lycée, parce qu'elles sont englobées dans une même temporalité de la journée lycéenne, moments de cours, pauses, moments de convivialité, dans ou en dehors de l'enceinte du lycée. Ainsi pendant les pauses :

« Où on va? Devant la route, des fois on s'assoit en bas, juste en bas du lycée... sinon, ben, on va au bar. Des fois on a rien à faire, on s'assoit vers le HLM. » (Elève de Première)

On peut remarquer également que les formes de transgression les plus banales, le cours « séché » ou le retard calculé, jouent avec cette règle temporelle.

Notre hypothèse est que ces pratiques, ces routines, ces rites permettent aux adolescents de s'approprier un espace, dont ils n'ont ni la maîtrise conceptuelle ni la maîtrise réglementaire, et un temps qui leur est imposé. Nous avons soulevé dans notre questionnement initial l'importance des règles d'usage des lieux et la diversité des temporalités coexistant dans l'établissement scolaire. Les routines et les rites élaborés par les élèves entre eux servent ainsi à organiser le partage de cet espace collectif et potentiellement conflictuel. Elles permettent également de se sécuriser: l'adolescent se trouve avec le passage au lycée dans une période de fragilisation, dont il cherche à se protéger en mettant en place des «stratégie de réassurance » (Duru-Bellat et Van Zanten, 1999), celles-ci sont généralement étudiées dans les comportements de groupe, mais ces usages des temps et des lieux peuvent également être interprétés à cette lumière. 


\section{Territorialité et usage des lieux}

Des territoires se dessinent au fil des récits, territoires instables, mouvants, territoires en archipel au sein desquels des lieux émergent avec force mais où l'on peine à identifier des liens. Réfléchir en termes de territorialité des lycéens nous paraît pertinent dans la mesure où l'on assiste à des processus d'appropriation des lieux, d'expérience de maîtrise d'espaces et de temps qui leur sont propres. Ce sont des processus en devenir, intrinsèquement liés à ces années de socialisation, à une identité transitoire mais profondément structurante de lycéen.

A travers les configurations que nous avons interprétées, les modalités de maîtrise de l'espace passent en grande partie par l'appropriation fragmentaire de lieux et de moments. Cette appropriation vise à «privatiser l'espace public » (Roché, 1996). Cet effort est une constante de notre rapport à l'espace. Chacun de nous tente d'instaurer des routines attachées à des lieux : s'asseoir toujours à la même place dans une salle, attendre le bus au même endroit, installer sa serviette sur le même coin de sable. Ces processus de territorialisation ont été largement décrits ailleurs; ils sont sans doute plus intenses et plus nécessaires pendant le tout jeune âge où l'élève doit faire son apprentissage du partage de l'espace : c'est le porte-manteau individuel à l'école, le casier; les nombreux « coins » aménagés dans les écoles maternelles témoignent de la prise en compte de ce besoin chez les plus petits. Nous avons eu l'occasion d'étudier dans d'autres cadres les modes d'appropriation individuels et collectifs de la cour de récréation à l'école primaire ${ }^{8}$. Les adolescents sont trop vite considérés comme affranchis de ces besoins ; on voit que ceux-ci prennent en fait des formes nouvelles beaucoup plus organisées en fonction du groupe : au besoin d'appropriation individuelle a succédé une appropriation par des collectifs à géométrie variable, du duo au groupe classe, aidé par une autonomie croissante. C'est en cela que le lycée nous apparaît comme un matériau sur lequel l'adolescent expérimente de nouvelles formes de territorialité. On a vu qu'il use de ce matériau de diverses manières : tantôt en surinvestissant un lieu matériellement identifié - le coin fumeur -, parfois mais plus rarement dans le cas que nous avons étudié en détournant l'usage d'un lieu - la rue devant le lycée -, parfois en ignorant la matérialité des lieux - l'enceinte du lycée. Il faudrait vraisemblablement rajouter à notre liste la transgression de la règle du lieu mais notre micro n'a pas recueilli, on s'en doute, ce type de confidence.

Pour l'élève, l'espace scolaire ne se réduit donc pas à la morphologie de l'établissement ni dans son extension (limites) ni dans son contenu, son épaisseur (l'ensemble des lieux qui s'y juxtaposent). Il y a des «en-deçà » et des « au-delà » de l'espace scolaire. Ils font naître un territoire scolaire propre à chaque élève et/ou à chaque groupe d'élèves. Ce territoire se réfère aux diverses pratiques de l'élève au sein de l'établissement mais aussi à l'extérieur de celui-ci, dans la mesure où ces pratiques sont exclusivement liées à l'école et aux relations sociales qui s'y fondent. Ce territoire s'appuie sur des constructions qui donnent corps et sens à la socialisation et à la territorialisation du monde par tout adolescent. Celles-ci sont faites de règles et de transgressions qui structurent la réalité territoriale du lycée, tant dans la dénomination, que dans l'utilisation des lieux. Cette réalité du lycée comme territoire scolaire relève de la composition plus ou moins structurée de lieux en lisière de l'établissement. En lisière seulement car l'ouverture au monde se fait prioritairement par contiguitté spatiale et sociale, celle des réseaux, des liens tissés. Cette contiguïté est juste suffisante pour symboliser la rupture sans tomber dans la coupure.

\footnotetext{
${ }^{8}$ Il s'agissait d'un mémoire de maîtrise sur l'occupation de la cour par les élèves de cours moyens (Miguet S. 2000) dont les acquis confirment notamment les travaux de l'anthropologue Julie Delalande (2001; 2003).
} 
De nombreux contacts avec des principaux, proviseurs, conseillers d'éducation nous ont montré que la question de la co-présence des divers membres de la communauté éducative, souvent conflictuelle, difficile, n'est jamais abordée en termes d'espace, de gestion et de partage de l'espace (Lapierre et Sgard, 2000). La réflexion se focalise sur les incivilités, les dégradations, le vandalisme, voire sur la violence scolaire si fréquemment médiatisée. Pour cela, elle se concentre quasi exclusivement sur la question des normes collectives au sein de l'établissement et cherche les solutions essentiellement dans le champ de la négociation et/ou de l'imposition de la norme, par le biais généralement du règlement intérieur, parfois renommé Charte.

Les études de S. Roché ont montré que, dans les collèges en particulier, les actes de vandalisme ou d'incivilité se déroulent fréquemment à des moments «creux » et dans les espaces intermédiaires, interstitiels, entre les salles de classe et les autres espaces aux fonctions clairement établies (CDI, cantine) : dans les couloirs, les escaliers, le garage à vélo, le hall. Ils sont sans surveillance mais surtout sans règle propre, sans responsabilité ni responsable affirmé, sans qualité, donc non appropriés (Roché, 2000).

Ce n'est pas tant sur le thème des incivilités et de la violence scolaire, que nous voulons nous situer, que sur la question de la qualification des espaces et de la prise en compte de cette qualification dans la recherche d'un espace négocié. En effet, si l'on accepte l'idée que l'adolescent use dans son apprentissage du temps social et de l'espace collectif et dans sa quête de socialisation, du temps et de l'espace qu'il a « sous la main »" l'école, le collège ou le lycée, pourquoi ne pas travailler dans le sens d'une objectivation de cet espacetemps : ses qualités matérielles, ses dimensions, ses règles, ses normes. Donner du sens à l'espace scolaire, réfléchir à un sens commun, amène les différents acteurs concernés à réfléchir sur leurs pratiques spatiales et leurs temporalités, leurs potentialités de combinaison et de cohabitation. Cela leur permet de se saisir de l'espace non comme un donné sur lequel on n'a pas prise, position habituelle des équipes pédagogiques et administratives, mais comme un outil, une ressource. Objectifs de responsabilisation, d'intégration dans un collectif et d'apprentissage de l'espace se trouvent ainsi combinés.

Les thématiques de l'appropriation et de l'intégration amènent à un questionnement qui, s'il n'est pas premier, sous-tend néanmoins notre démarche, celui de la construction des identités par les adolescents. Celle-ci est délicate à analyser et notre matériau ne nous permet pas de mener une interprétation très approfondie; nous nous contenterons donc d'ouvrir quelques pistes d'exploration. Comme nous l'avons dit, le lycée est une étape transitoire mais marquante de la vie d'adolescent, surtout s'il reste toute sa scolarité dans le même établissement; s'il consomme de l'espace sans vraiment lui donner sens, il se construit une identité lycéenne avant tout nourrie de relations sociales, avec ses pairs, avec les adultes. La plupart des pratiques qu'il a pu élaborer pendant cette période disparaissent dès le lycée quitté, souvent les réseaux d'amitié se dispersent, une page est tournée. On serait donc tenté de conclure à la faible place du lycée dans la construction d'une identité et de surcroît d'une identité territoriale. Néanmoins, par les diverses formes d'expérimentation que nous avons montrées, la vie lycéenne est le moment des apprentissages, des initiations, des premières constructions territoriales relativement autonomes. Le lycée peut donner prise à la construction d'un sentiment d'appartenance à un groupe et à un territoire, pour la première fois extérieurs à la famille ; c'est du reste ce que tentent d'impulser de nombreuses équipes de direction. Par ailleurs, de cette période, les anciens élèves que nous avons interrogés gardent une mémoire, tantôt nostalgique, tantôt désagréable, toujours exprimée, dans laquelle le lycée est bien présent mais sous une forme revisitée : ils accordent plus de place au bâti, aux impressions sensibles restées en mémoire : des bruits, des odeurs. La

\footnotetext{
${ }^{9}$ L'utilisation de l'espace-classe par les élèves de maternelle montre que les plus petits usent spontanément de cet outil pour expérimenter leur rapport à l'espace : distance, proximité, partage.
} 
mémoire tend ainsi à reconfigurer les lieux, outrant parfois le trait, replaçant ce lieu chargé de souvenirs dans leur territoire d'étudiant: ils ne s'y rendent généralement plus, mais aucun n'a manifesté la volonté de le gommer de ses souvenirs.

Au cours de cette recherche, nous avons voulu mener une démarche de coconstruction de cet objet incertain, nommé dans un premier temps espace scolaire, puis plus simplement lycée, avec les nuances et modalités d'usages que nous avons relevées au fil des entretiens, pour l'intégrer enfin dans une analyse des territorialités en devenir des élèves. A travers ces rencontres avec les élèves, nous avons cherché à les intégrer dans une démarche tant exploratoire que participative : expliquer nos objectifs, expliciter notre questionnement et finalement construire au cours d'un constant va-et-vient entre hypothèses de départ et interprétation des entretiens - que ce texte par souci de clarté ne retranscrit pas - la caractérisation et l'analyse. Cette démarche répond autant à un souci de chercheur : construire l'objet avec les acteurs, que de pédagogue : conduire les élèves à une réflexion sur leurs usages et leurs pratiques de l'espace. Si tant est que l'on puisse ici distinguer deux versants de la démarche. Car comme le rappelle justement B. Mérenne-Schoumaker, « les recherches récentes sur les apprentissages montrent bien l'importance de la motivation et de l'intérêt dans la construction de nouvelles connaissances. Rappelons que la motivation est de l'ordre de l'affectif, du désir ou plus simplement de l'envie d'apprendre alors que l'intérêt est de l'ordre du sens. Tous deux se rejoignent souvent et sont fondamentaux pour «l'autosocioconstruction » des savoirs, c'est-à-dire la théorie selon laquelle tout savoir est une construction du sujet en réponse aux sollicitations de l'environnement » $(2002,121)$.

Cette démarche de construction des savoirs sur l'espace à partir d'une analyse des constructions territoriales de l'élève par l'élève souhaiterait trouver un prolongement didactique. Et cela même si les programmes de géographie de collège et lycée laissent peu de place à l'espace du quotidien, au territoire individuel, à la réflexion des élèves sur leurs propres constructions. Il nous semble en effet que les adolescents sont volontiers interpellés par ce renversement du regard qui leur est ici proposé, et sont susceptibles de s'emparer de cette ressource, l'espace scolaire. Si la Géographie enseignée répond difficilement à ces objectifs, l'éducation civique, l'ECJS, la formation des élèves délégués, voire les TPE, peuvent constituer des alliés intéressants.

Ce champ intéresse la géographie dans la mesure où ses objets et catégories traditionnels se sont trouvés interrogés : tantôt opératoires (le lieu), tantôt à reformuler (les limites), tantôt non pertinents (espace). Il nous semble en tout cas que la géographie est à même d'apporter des outils d'analyse nouveaux et utiles aux acteurs du système éducatif : à I'heure où se pose quotidiennement la question de la gestion des établissements, déplacer le point de vue de l'institution ou de la communauté éducative vers les usagers des lieux, peut permettre de renouveler les termes du débat. Les expressions qui apparaissent, telle la récente «Maison du lycéen », qui remplace l'ancien «foyer», montrent bien la dimension « habitante » de l'adolescent dans son métier d'élève. Il reste à saisir cette dimension dans toute sa richesse mais cela demande une réflexion légèrement décentrée par rapport à l'objectif que nous nous sommes fixé dans cet article. En effet, au vu des entretiens, cette dimension va bien au-delà du simple territoire scolaire, même si celui-ci au même titre que le territoire domestique est essentiel à la structuration de l'adolescent. Il sera donc intéressant pour nous de poursuivre cette démarche vers une meilleure explicitation du monde de l'adolescent dans ses multiples caractéristiques spatiales, sociales et temporelles, et d'entrevoir peut-être l'ensemble des territoires que chaque élève nourrit de ses investissements, de ses réalisations, de ses déceptions aussi. 


\section{Bibliographie}

ANDRÉ Y. (1989a), « Lire et dire l'espace - L'utilisation des représentations pour un apprentissage à la lecture et à la maîtrise de l'espace - $\gg$, dans Y. André et al., Représenter l'espace: L'imaginaire spatial à l'école, Paris, Anthropos-Economica, p. 125-140.

ANDRÉ Y. (1989b), « Les cartes mentales », dans Y. André et al., Représenter l'espace : L'́maginaire spatial à l'école, Paris, Anthropos-Economica, p. 153-167.

AUDIGIER F. (1990), « Représentations des élèves et didactique de la géographie : Exemple de la ville », Talence-Bordeaux, CEGET-CNRS, Colloque Ethnographie.

AUGOYARD J.-F. (1979), Pas à pas. Essai sur le cheminement quotidien en milieu urbain, Paris, Editions du Seuil.

BIACHE C. (1997), «La ville et la banlieue. Représentations d'enfants de CM1 et CM2 », dans C. Callenge, M. Lussault et B. Pagand (dir.), Figures de l'urbain. Des villes, des banlieues et de leurs représentations, Tours, Maison des Sciences de la Ville, p. 165-178.

BOUJU A. (1997), « De la dimension spatiale du champ scolaire: Représentations et pratiques du territoire liées à la scolarisation dans les espaces périurbains », dans C. Callenge, $M$. Lussault et B. Pagand (dir.), Figures de l'urbain. Des villes, des banlieues et de leurs représentations, Tours, Maison des Sciences de la Ville, p. 205-213.

CALOGIROU C. (1991), « De l'influence du lieu sur les rapports microsociaux. Ses conséquences sur la socialisation des jeunes », dans H. Malewska-Peyre et P. Tap (dir.), La socialisation de l'enfance à l'adolescence, Paris, PUF, p. 219-232.

CAVALLINI V. (1997), «La perception de la ville de Cergy-Pontoise par des élèves de seconde du Lycée Alfred-Kastler (1990-1994) », dans C. Callenge, M. Lussault et B. Pagand (dir.), Figures de l'urbain. Des villes, des banlieues et de leurs représentations, Tours, Maison des Sciences de la Ville, p. 131-149.

CHEVALIER J.-P. (1997), « Géographie de l'Ecole et géographie à l'Ecole : Etude du fichier des thèses de 1990 à $1994 \gg$, Cybergéo: Revue Européenne de géographie, $n^{\circ}$ 24, http://193.55.107.3/didact/texte/geodidac.htm.

CHOMBART DE LAUWE M.-J. (1987), Espaces d'enfants, la relation enfant-environnement, ses conflits, Lausanne, Editions Delval.

CLERC P. (1997), «Les images de la ville dans les manuels scolaires. Une image de la géographie », dans C. Callenge, M. Lussault et B. Pagand (dir.), Figures de l'urbain. Des villes, des banlieues et de leurs représentations, Tours, Maison des Sciences de la Ville, p. 119-129.

DEBARBIEUX B., FOURNY M.-C. (dir.) (2004), L'effet géographique. Construction sociale, appréhension cognitive et configuration matérielle des objets géographiques, Grenoble, Editions de la MSH-Alpes.

DEBARBIEUX E. (dir.) (2000), La violence en milieu scolaire, Paris, ESF Editeur, coll. « Actions sociales/Confrontations 》, 2 tomes.

DELALANDE J. (2001), La cour de récréation : Contribution à une anthropologie de l'enfance, Rennes, Presses Universitaires de Rennes.

DELALANDE J. (2003), La récré expliquée aux parents : De la maternelle à l'école élémentaire. La vie quotidienne dans une cour d'école, Paris, Louis Audibert Editions.

DEROUET-BESSON M.-C. (1998), Les murs de l'école. Eléments de réflexion sur l'espace scolaire, Editions Métailié.

DI MEO G.(dir.) (1996), Les territoires du quotidien, Paris, L'Harmattan.

Di MEO G. (1998), Géographie sociale et territoires, Paris, Editions Nathan.

DUBET F. (1991), Les lycéens, Paris, Seuil.

DURU-BELLAT M. et HENRIOT - VAN ZANTEN A. (1998), Sociologie de l'école, Paris, Armand Colin.

GAYET D. (2003), L'élève, côté cour, côté classe, Paris, INRP.

GIRAUD B. (1989), « Un exemple élémentaire d'emploi de la géographie des représentations en classe de collège » dans Y. André et al., Représenter l'espace : L'imaginaire spatial à l'école, Paris, Anthropos-Economica, p. 191-196.

GUÉRIN M.-C. (1997), «Enseigner les villes américaines », dans C. Callenge, M. Lussault et B. Pagand (dir.), Figures de l'urbain. Des villes, des banlieues et de leurs représentations, Tours, Maison des Sciences de la Ville, p. 151-156.

HAICAULT M. (1989), «Enfants et temps quotidien: apprentissages et transmissions », dans "Temps scolaires et socialisation", Temporalistes, $n^{\circ} 10$, p. 5-10. 
HENRIOT - VAN ZANTEN A. (1990), L'école et l'espace local : les enjeux des zones d'éducation prioritaires, Lyon, Presses Universitaires de Lyon.

HENRIOT - VAN ZANTEN A. (dir.) (2000), L'école : L'état des savoirs, Paris, Editions La Découverte.

HERIN R., ROUAULT R. et VESCHAMBRE V. (dir.) (1994), Atlas de la France scolaire de la maternelle au lycée, Montpellier / Paris, GIP Reclus / La Documentation Française.

HOYAUX A.-F. (2003), «Les constructions des mondes de I'habitant: Eclairage pragmatique et herméneutique », Cybergéo: Revue Européenne de Géographie, $n^{\circ}$ 232, http://www.cybergeo.presse.fr/ehgo/hoyaux/hoyaux203.htm.

LAPIERRE G. et SGARD A. (dir.) (2000), De l'incivilité à la conscience citoyenne : construire l'éducation à la citoyenneté dans l'établissement scolaire, Grenoble, Université Pierre Mendès-France, Plan national de formation 1999-2000.

LÉVY J. (2003), « Espace Public », dans J. Lévy et M. Lussault (dir.), Dictionnaire de la Géographie et de l'espace des sociétés, Paris, Belin, p. 336-339.

LUGADET-AGRAZ J.-B. (1989), Représentations enfantines de l'espace urbain: Exemples clermontois », dans Y. André et al., Représenter l'espace : L'ímaginaire spatial à l'école, Paris, Anthropos-Economica, p. 179-190.

LUSSAULT M. (2003), «Espace Public », dans J. Lévy et M. Lussault (dir.), Dictionnaire de la Géographie et de l'espace des sociétés, Paris, Belin, p. 333-336.

MALEWSKA-PEYRE H. et TAP P. (dir.) (1991), La socialisation de l'enfance à l'adolescence, Paris, PUF.

MANGIN C. (1997), « Nancy des riches, Nancy des pauvres : des collégiens se représentent leur ville $\gg$, Mappemonde, $n^{\circ} 2$, p. 35-40.

MASSON M. (1995), L'enfant et la montagne. Savoirs géographiques et représentations spatiales sur la montagne, Paris, Anthropos-Economica.

MÉRENNE-SCHOUMAKER B. (2002), Analyser les territoires. Savoirs et outils, Rennes, Presses Universitaires de Rennes.

MIGUET S. (2001), Représentation spatiale des enfants : L'appropriation de la cour de récréation par les élèves, Grenoble, Université Joseph Fourier, Mémoire de Maîtrise.

MOLES A., ROHMER E. (1978), Psychologie de l'espace, Paris - Tournai, Casterman.

PARTOUNE C. (1999), Quelles compétences terminales dans l'enseignement de la géographie?, Ministère de la Communauté Française de Belgique, Département de l'Education, de la Recherche et de la Formation, Pilotage de l'enseignement inter-réseau, Recherche en éducation $\mathrm{n}^{\circ} 12 / 97$, http://www.ulg.ac.be/geoco//mg/competences/chantier.

PIAGET J., INHELDER B. (1948), La représentation de l'espace chez l'enfant, Paris, PUF.

PIAGET J. (1981), Le développement de la notion de temps chez l'enfant, Paris, PUF, $3^{\text {ème }}$ éd.

RAYON P. (1998), La cité des lycéens, Paris, L'Harmattan.

ROCHÉ S. (1996), La société incivile. Qu'est-ce que l'insécurité ?, Paris, Seuil.

ROCHÉ S. (2000), « L'accord sur les incivilités », dans G. Lapierre et A. Sgard (dir.), De l'incivilité à la conscience citoyenne: construire l'éducation à la citoyenneté dans l'établissement scolaire, Grenoble, Université Pierre Mendès-France, Plan national de formation 1999-2000, p. 31-43.

ROCHÉ S. et SCHLEGEL J.-L. (2000), La société d'hospitalité, Paris, Seuil.

SAARINEN T. (1989), «Images du Monde à travers les cartes mentales », dans Y. André et al., Représenter l'espace : L'imaginaire spatial à l'école, Paris, Anthropos-Economica, p. 169-177.

SENORE D. (2000), L'aménagement des temps et des espaces, Paris, INRP.

TAP P. (1991), « Socialisation et construction de l'identité personnelle », dans H. Malewska-Peyre et P. Tap (dir.), La socialisation de l'enfance à l'adolescence, Paris, PUF, p. 219-232. 